\title{
Is meconium exposure associated with autism spectrum disorders in children?
}

\author{
Ensiyeh Jenabi, PhD ${ }^{1}$, Erfan Ayubi, $\mathrm{PhD}^{2,3}$, Salman Khazaei, $\mathrm{PhD}^{4}$, Saeid Bashirian, PhD ${ }^{5}$, Mojtaba Khazaei, MD \\ ${ }^{1}$ Autism Spectrum Disorders Research Center, Hamadan University of Medical Sciences, Hamadan, Iran; ${ }^{2}$ Health Promotion Research Center, Zahedan University of \\ Medical Sciences, Zahedan, Iran; ${ }^{3}$ Department of Community Medicine, School of Medicine, Zahedan University of Medical sciences, Zahedan, Iran; ${ }^{4}$ Research Center \\ for Health Sciences, Hamadan University of Medical Sciences, Hamadan, Iran; ${ }^{5}$ Social Determinants of Health Research Center, Hamadan University of Medical \\ Sciences, Hamadan, Iran; ${ }^{6}$ Department of Neurology, School of Medicine, Hamadan University of Medical Sciences, Hamadan, Iran
}

Background: The results differ among published studies regarding exposure to meconium and the risk of developing autism spectrum disorders (ASDs).

Purpose: The present study pooled all of the epidemiologic studies retrieved from broader databases on the association between meconium exposure and risk of developing ASD in children.

Methods: The Web of Science, PubMed, Scopus, and Google Scholar databases were searched without language restrictions for articles published between their inception to February 20, 2020, using relevant keywords. The pooled odds ratios (ORs) and their $95 \%$ confidence intervals (CIs) were calculated as random-effect estimates of the associations among studies. A subgroup analysis was conducted to explore any potential sources of heterogeneity among studies.

Results: The pooled estimate of OR reported a weakly sig. nificant association between meconium exposure and ASD development in children (OR, 1.13; 95\% CI, 1.03-1.24). There was low heterogeneity among the articles reporting risk for ASD among children $\left(I^{2}=19.3 \% ; P=0.259\right)$. The results of subgroup analysis based on meconium exposure showed a significant association between a meconium-stained neonate and ASD development (OR, 1.18; 95\% CI, 1.11-1.24).

Couclusion: Meconium exposure was weakly associated with an increased risk of ASD. However, more evidence based on large prospective cohort studies is required to provide conclusive evidence about whether meconium exposure is associated with an increased risk of ASD development.

Key words: Autism spectrum disorder, Meconium, Metaanalysis

\section{Key message}

- Meconium exposure is weakly associated with an increased risk of autism spectrum disorder (ASD) development in children.
- More evidence based on large prospective cohort studies is required to conclude whether meconium exposure is associated with an increased risk of ASD.

\section{Introduction}

Autism spectrum disorders (ASDs) are a complex of chronic neurological situations consisting of persistent shortfalls in social communication, repetitive behaviors or activities in childhood, and inappropriate behavior. ${ }^{1)}$ The prevalence of ASDs has increased in recent years, especially in developed countries. ${ }^{2)}$ Evidence shows that the incidence of ASD has increased more than 10 -fold in the last 3 decades. ${ }^{3)}$ The increased survival rate of infants in neonatal intensive care units may partly justify the increased incidence of ASDs. ${ }^{4}$

Although the etiology of ASD is not fully understood, the role of heritability as well as some gestational environmental risk factors, including gestational age, infection, micronutrient insufficiency, fetal hypoxia, and stress, has been somewhat confirmed through various studies. ${ }^{5-9)}$ However, for variables whose effects on ASD remain unclear, meta-analyses remain the gold standard.

The passage of meconium prior to delivery, which occurs in 5\%-25\% of term births, causes nearly $5 \%$ of neonates to develop meconium aspiration syndrome (MAS). ${ }^{10)}$ MAS can increase the rate of infection or asphyxia in neonates. ${ }^{11)}$ The effect of MAS on the development of cerebral palsy or neurodevelopmental delay has previously been described. ${ }^{12)}$ Beligere and Rao ${ }^{13)}$ suggested that infants with a diagnosis of MAS later manifest neurodevelopmental delays, even if they respond well to conventional treatment.

Several studies have been published regarding exposure to meconium and the risk of developing ASD..$^{1,4,14-21)}$ However, their results differed; therefore, the present systematic review and meta-analysis pooled all epidemiologic case-control and cohort studies retrieved from broader databases on the association between meconium exposure and the risk of developing of ASD in children.

Corresponding author: Mojtaba Khazaei, MD. Sina Hospital, Hamadan University of Medical Sciences, Hamadan, Iran

凶 Email: khazaeimojtaba@yahoo.com, https://orcid.org/0000-0002-2360-9260

Received: 15 June, 2020, Revised: 25 July, 2020, Accepted: 12 August, 2020

This is an open-access article distributed under the terms of the Creative Commons Attribution Non-Commercial License (http://creativecommons.org/licenses/by$\mathrm{nc} / 4.0 /$ ) which permits unrestricted non-commercial use, distribution, and reproduction in any medium, provided the original work is properly cited.

Copyright (c) 2021 by The Korean Pediatric Society 


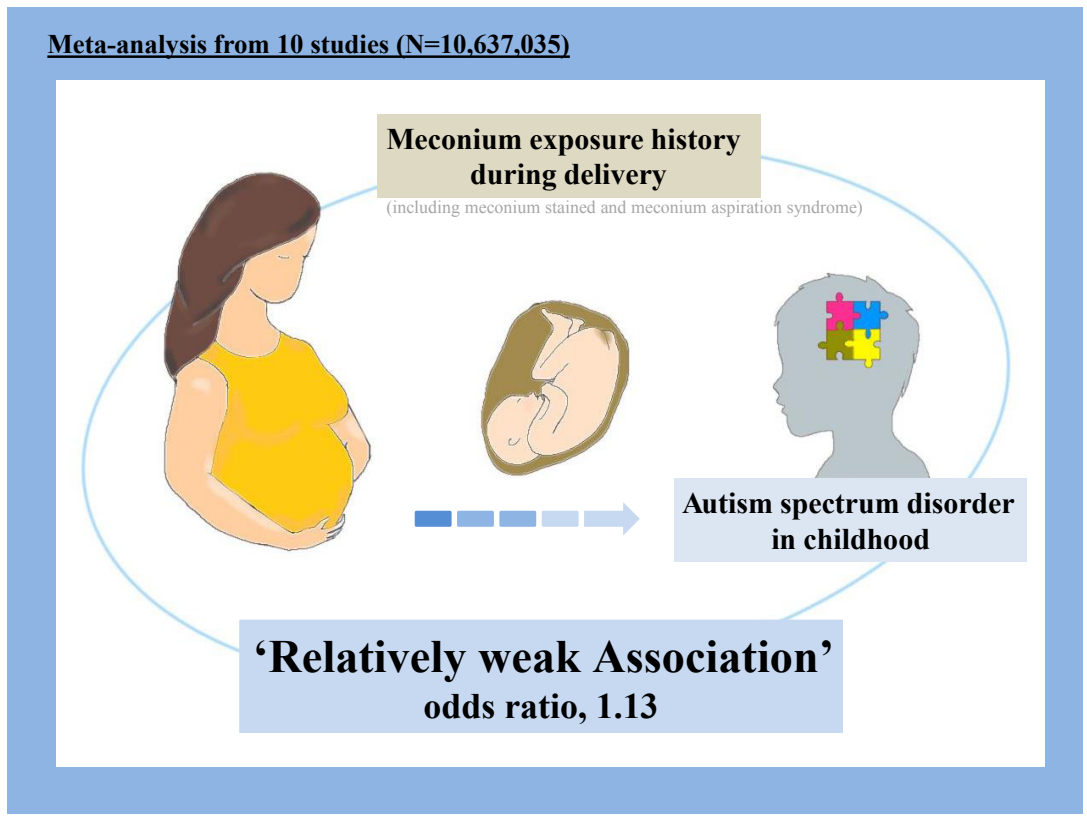

Graphical abstract

\section{Methods}

\section{Data sources and search strategy}

This meta-analysis aimed to assess of the association between meconium exposure and risk of developing of ASD in children. We performed the systematic review using the published PRISMA (Preferred Reporting Items for Systematic Reviews and MetaAnalyses) statement checklist. The Web of Science, PubMed, Scopus, and Google Scholar databases were searched without language restrictions for relevant articles published between inception and February 20, 2020.

Medical Subject Headings keywords and terms including "meconium-stained liquor," "meconium-stained," "meconium aspiration syndrome," "meconium," "not clear amniotic fluid," "ASD," "autism spectrum disorder," and "autism spectrum disorders," with the help of Boolean operators (AND or OR), were used individually or in combination to ensure a comprehensive search (Supplement material 1).

\section{Inclusion and exclusion criteria}

Case-control and cohort studies reporting the association between meconium exposure and the development of ASD in children were included in the present meta-analysis. Case reports, letters to the editor, systematic reviews, and meeting abstracts were excluded. Duplicate publications were also excluded. In addition, to decrease the risk of error, all processes were independently completed by 2 reviewers (EJ and SK).

\section{Data extraction}

The data from each study were recorded in a data extraction form designed by the researchers. The retrieved data included: the first author's name, year of publication, country, study design, exposure type, adjustment, autism criteria, and study quality.

\section{Meta-analysis}

The data analysis was conducted using Stata 13 (Stata Corp., College Station, TX, USA). The pooled odds ratios (ORs) and their 95\% confidence intervals (CIs) were calculated. The randomeffects model was used for the meta-analysis. Subgroup analyses were conducted based on the adjusted studies, meconium exposure type, and study design to identify potential sources of heterogeneity. The statistical heterogeneity among the studies was determined using the Cochrane $\mathrm{Q}$ and $\mathrm{I}^{2}$ tests. To evaluate possible publication bias, the quantitative Egger weighted regression test was applied considering values of $P<0.05$ as statistically significant. A funnel plot was used to visually determine publication bias. Furthermore, the study distribution was assessed using Egger linear regression test.

\section{Quality assessment}

We used the improved Newcastle-Ottawa scale (NOS) to assess the quality of the included studies. The scale includes participant selection, comparability of the children with versus those without ASDs, and outcome assessments. Two investigators conducted the assessment independently and scored the studies as low quality ( $<7$ points) or high quality ( $\geq 7$ points).

\section{Results}

\section{Study description}

A total of 366 studies were retrieved in the initial search. Of them, we excluded 152 duplicates; thus, 214 were subjected to title, abstract, and full-text review. A total of 195 studies were excluded after title and abstract screening, while 19 studies were subjected to full-text review. We excluded 9 studies for not meeting the inclusion criteria (review and meta-analyses). A total 
Identification

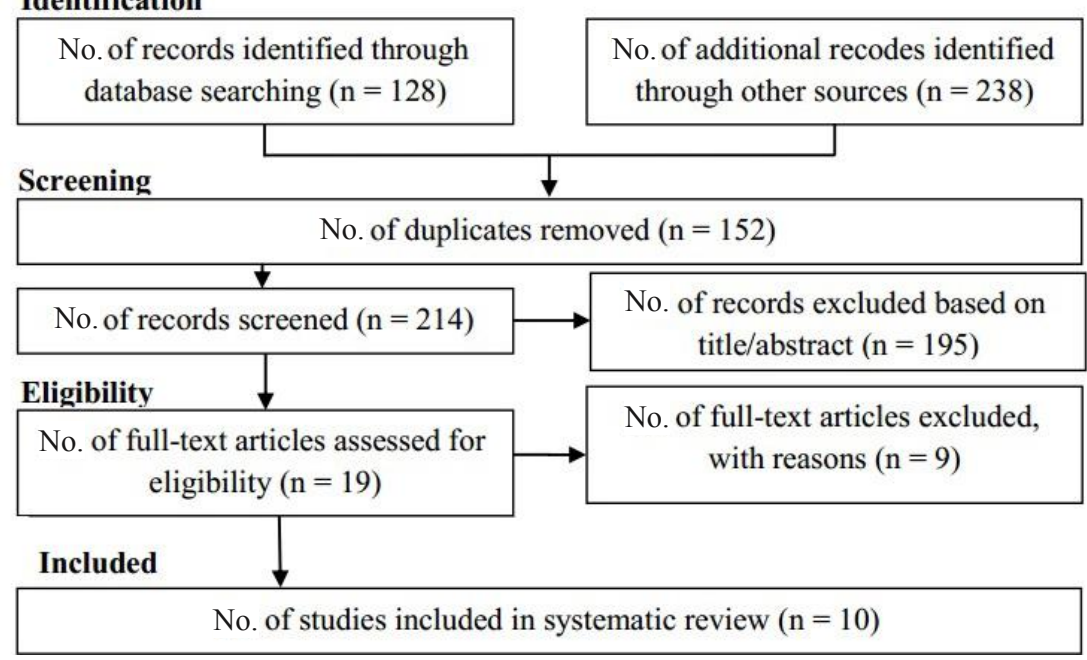

Fig. 1. Diagram of the different phases of the systematic review.

\begin{tabular}{l} 
Study \\
ID \\
\hline Matsuishi, 1999 (95\% Cl)
\end{tabular}

Fig. 2. Forest plot of the association between meconium and autism spectrum disorder development in children.

of 10 studies were included in the meta-analysis. The study selection process is shown in Fig. 1. The studies included 5 cohort studies ${ }^{1,18-21)}$ and 5 case-control studies, ${ }^{4,14-17)}$ with a total sample size of 10,637,035 participants.

The association between meconium exposure and ASDs in children had confounding variables including mother's and father's age, race, parity, obesity, birth weight, gestational age, Apgar score, birth defects, presentation, mode of delivery, and child's sex.

\section{Main analysis}

Fig. 2 shows the association between meconium exposure and ASDs in children. The pooled OR estimate reported a significant association between meconium exposure and ASDs in children (OR, 1.13; 95\% CI, 1.03-1.24). There was low heterogeneity among the studies reporting risk of ASD development in children $\left(\mathrm{I}^{2}=19.3 \% ; P=0.259\right)$.

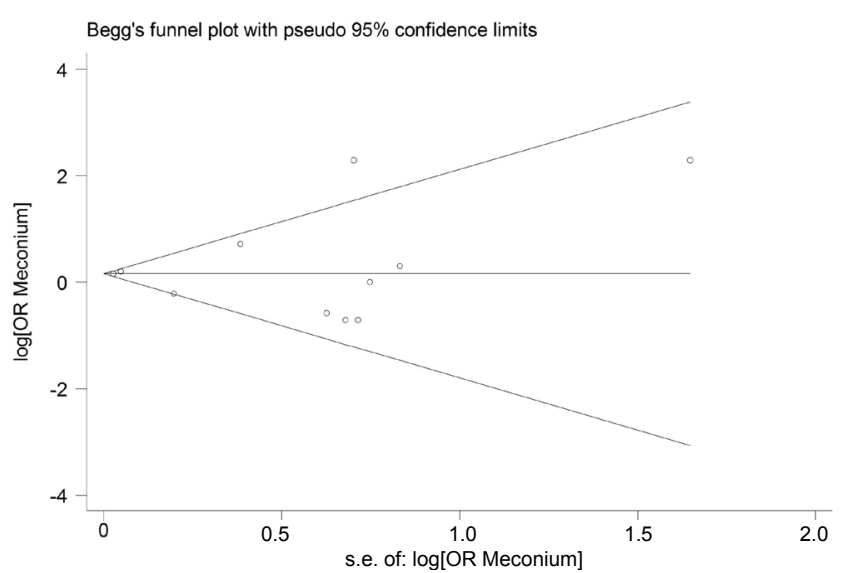

Fig. 3. Funnel plot of the association between meconium and autism spectrum disorder development in children. OR, odds ratio. 
Table 1. Summary of the included studies

\begin{tabular}{|c|c|c|c|c|c|c|c|c|c|}
\hline Study & Country & Design & Exposure & Sample size & Estimate & Adjustment & Autism criteria & Child age (yr) & Quality \\
\hline Piven et al. ${ }^{16)}$ (1993) & USA & Case-control & MS & 78 & Odds ratio & Crude & ICD-10 & 14.5 & Low \\
\hline Matsuishi et al. ${ }^{15)}$ (1999) & Japan & Case-control & MAS & 5,271 & Odds ratio & Crude & DSM & $2-5$ & Low \\
\hline Maimburg and Vaeth ${ }^{14)}(2006)$ & Denmark & Case-control & M & 922 & Odds ratio & Adjusted & ICD-8; ICD-10 & 4.57 & High \\
\hline Bilder et al. ${ }^{18)}(2009)$ & USA & Cohort & M & 26,315 & Odds ratio & Adjusted & DSM & Not reported & High \\
\hline Nath et al. ${ }^{4)}$ (2012) & India & Case-control & MAS & 131 & Odds ratio & Crude & DSM & 3 & High \\
\hline Gregory et al. ${ }^{19)}$ (2013) & USA & Cohort & M & 625,042 & Odds ratio & Adjusted & Not reported & Not reported & High \\
\hline $\begin{array}{l}\text { Mrozek-Budzyn et al. }{ }^{17)} \\
\text { (2013) }\end{array}$ & Poland & Case-control & MS & 288 & Odds ratio & Crude & Medical record & $2-15$ & High \\
\hline $\begin{array}{l}\text { Winkler-Schwartz et al. }{ }^{21)} \\
(2014)\end{array}$ & Canada & Cohort & MS/MAS & 180 & Odds ratio & Crude & DSM & 7.8 & High \\
\hline Miller et al. ${ }^{1)}(2017)$ & Canada & Cohort & M/MS/MAS & $9,945,896$ & Odds ratio & Adjusted & ICD-9 & $4+$ & High \\
\hline Wu et al. ${ }^{20)}(2017)$ & China & Cohort & M & 32,912 & Odds ratio & Crude & DSM; ADI-R & 3 & High \\
\hline
\end{tabular}

M, meconium; MS, meconium-stained; MAS, meconium aspiration syndrome; ICD-8, International Classification of Diseases version 8; ICD-10, International Classification of Diseases version 10; DSM, Diagnostic and Statistical Manual of Mental Disorders; ADI-R, Autism Diagnostic Interview-Revised

Table 2. Results of subgroup analysis of the association between meconium exposure and the development of ASD development based on ORs

\begin{tabular}{lccr}
\hline \multirow{2}{*}{ Subgroups } & \multicolumn{3}{c}{ Studies } \\
\cline { 2 - 4 } & No. of studies & OR $(95 \% \mathrm{Cl})$ & \multicolumn{1}{c}{$\mathrm{I}^{2}$} \\
\hline Crude/adjusted analysis & & $0.79(0.20-1.39)$ & $0 \%$ \\
$\quad$ Crude & 6 & $1.13(1.01-1.26)$ & $57.7 \%$ \\
$\quad$ Adjusted & 4 & & \\
Study design & & & \\
$\quad$ Case-control & 5 & $0.86(0.52-1.20)$ & $0 \%$ \\
Cohort & 5 & $1.16(1.07-1.26)$ & $39.0 \%$ \\
\hline
\end{tabular}

ASD, autism spectrum disorder; OR, odds ratio; $\mathrm{Cl}$, confidence interval.

No publication bias was identified by the Begg or Egger tests ( $P=0.392$ and $P=0.877$, respectively) (Fig. 3).

\section{Study quality}

The present meta-analysis included 2 low-quality studies and 8 high-quality studies according to the NOS scale score (Table 1 ).

\section{Subgroup meta-analysis}

We performed the subgroup analysis by results type (crude/ adjusted) and study design. The pooled results based on OR of the crude and adjusted studies were $0.79(0.20-1.39)$ and 1.13 (1.01-1.26), respectively. There was a significant association in the adjusted studies. The pooled results based on OR in the case-control and cohort studies were $0.86(0.52-1.20)$ and 1.16 (1.07-1.26), respectively. There was a significant association in the cohort studies (Table 2).

\section{Discussion}

Overall, the meta-analysis of adjusted ORs demonstrated that risk of ASD increased by $13 \%$ among children exposed to meconium in comparison to unexposed children. Upon stratification, meconium exposure was significantly associated with a 1.18- fold increased risk of ASD. Moreover, meconium exposure was significantly associated ( $16 \%$ increased risk) with ASD in children in a meta-analysis of the cohort studies.

The sum of the weights of the individual cohort studies in this meta-analysis was more than $90 \%$. Cohort studies can be considered the "gold standard" among observational studies since temporality from exposure to outcomes can be easily established (e.g., children exposed to meconium are followed until ASD does or does not occur). However, observational studies are prone to selection bias, information bias, and confounding factors; as a result, the meta-analysis results of such studies should be interpreted with caution. For example, Bilder et al. ${ }^{18)}$ mentioned that results may be affected by selection bias as a result of the total migration out of state and within the study area. In another included study ${ }^{19)}$ the authors mentioned that all potential confounders were not considered in the causal pathway for the incidence of ASD, so a degree of residual confounding remains in the resulting effect estimates. Moreover, Miller et al. ${ }^{1)}$ reported a degree of misclassification in the outcome of interest. Considering the above issues, the pooled association of 1.13 may differ from the true value.

It should be noted that the estimated pooled association of 1.13 may not be generalizable to all developmental disorders considered ASDs such as autistic disorder or pervasive developmental disorder-not otherwise specified. For example, the metaanalysis of Gardener et al. ${ }^{22}$ examined the effect of perinatal and neonatal factors, including meconium exposure, on the development of autism, the most common ASD, and demonstrated that meconium aspiration was significantly associated with a 7.34fold increased risk of autism, while the summary effect estimate for meconium staining was 0.82 , not statistically significant. Here, it should be considered that the used search strategy and databases in the aforementioned meta-analysis ${ }^{22}$ differed from those used in present study.

In the present meta-analysis, the risk of ASD was assessed separately by type of meconium exposure. MAS, as a severe form of meconium exposure, results in an $8 \%$ increase in the risk of 
ASD and was not statistically significant, whereas the milder form of meconium staining was the only type associated with an increased risk of ASD. The possible explanation for this is that neonates with MAS typically receive oxygen therapy or other treatments that may decrease the potential for brain damage ${ }^{23)}$; on the other hand, neonates with meconium-stained amniotic fluid are deprived oxygenation benefit, making obstruction of the airways and profound hypoxia more common.

Regardless of ASD status, the association between meconium exposure and other types of neurodevelopmental disorders, such as cerebral palsy, learning disabilities, and intellectual disabilities, has been noted in the literature. ${ }^{24)}$ Other complications associated with meconium exposure such as hypoxic-ischemic encephalopathy ${ }^{25)}$ or arterial ischemic stroke ${ }^{26)}$ were also reported. Hypoxia can delay the maturation of GABAergic neurons in the cerebral cortex and lead to neuron deregulation. ${ }^{27)}$ The dose, duration (acute or chronic), and degree (mild or severe) of fetal hypoxia determines the form and extent of neurodevelopmental impairment. Many studies have reported that children with ASD have disturbed neuronal overgrowth in regions of the brain. ${ }^{28)}$ Further large-sample cohort studies are needed to evaluate the effect of meconium exposure on long-term morbidities.

This meta-analysis has some limitations that should be considered. The main limitation is the small number of eligible studies included; as such, other articles from other databases, grey literature, or local documents may have been ignored. Another limitation was a lack of available data for the subgroup analysis by some variables such as mode of delivery and mode of treatment. Furthermore, there was great variation in the sample sizes of the included studies (e.g., one had a sample size of 78, while another had over 9 million), which yielded some bias in the results, such as small study effects.

In conclusion, the results of this meta-analysis showed that meconium exposure is weakly associated with an increased risk of the development of ASD. Addressing the effect of meconium exposure on short- and long-term adverse outcomes requires further well-designed and large-sample cohort studies. However, more evidence from large prospective cohort studies is required to provide conclusive evidence about whether meconium exposure is associated with an increased risk of ASD.

\section{Conflicts of interest}

No potential conflict of interest relevant to this article was reported.

\section{Acknowledgments}

Hamadan University of Medical Sciences financially supported this study but had no role in the data analysis and interpretation process, writing of the manuscript, or decision to submit the manuscript for publication. The protocol of this study was confirmed by Hamadan University of Medical Sciences with Code 99010537.
Supplementary material

Supplementary material can be found via https://doi.org/10. 3345/cep.2020.01053.

See the commentary "Recent Trends in Autism Spectrum Disorder Research using Text Mining of PubMed: Importance of Early Detection" via https://doi.org/10.3345/cep.2020.01564.

\section{References}

1. Miller KM, Xing G, Walker CK. Meconium exposure and autism risk. J Perinatol 2017;37:203-7.

2. Rai D, Lee BK, Dalman C, Golding J, Lewis G, Magnusson C. Parental depression, maternal antidepressant use during pregnancy, and risk of autism spectrum disorders: population based case-control study. BMJ 2013;346:£2059.

3. Fombonne E. Epidemiology of pervasive developmental disorders. Pediatr Res 2009;65:591-8.

4. Nath S, Roy R, Mukherjee S. Perinatal complications associated with autism--a case control study in a neurodevelopment and early intervention clinic. J Indian Med Assoc 2012;110:526-9.

5. Chaste P, Leboyer M. Autism risk factors: genes, environment, and geneenvironment interactions. Dialogues Clin Neurosci 2012;14:281-92.

6. Moore GS, Kneitel AW, Walker CK, Gilbert WM, Xing G. Autism risk in small- and large-for-gestational-age infants. Am J Obstet Gynecol 2012; 206:314.e1-9.

7. Harrington RA, Lee LC, Crum RM, Zimmerman AW, Hertz-Picciotto I. Prenatal SSRI use and offspring with autism spectrum disorder or developmental delay. Pediatrics 2014;133:e1241-8.

8. Walker CK, Krakowiak P, Baker A, Hansen RL, OzonoffS, Hertz-Picciotto I. Preeclampsia, placental insufficiency, and autism spectrum disorder or developmental delay. JAMA Pediatr 2015;169:154-62.

9. Krakowiak P, Walker CK, Bremer AA, Baker AS, Ozonoff S, Hansen RL, et al. Maternal metabolic conditions and risk for autism and other neurodevelopmental disorders. Pediatrics 2012;129:e1121-8.

10. Fanaroff AA. Meconium aspiration syndrome: historical aspects. J Perinatol 2008;28 Suppl 3:S3-7.

11. Monen L, Hasaart TH, Kuppens SM. The aetiology of meconium-stained amniotic fluid: pathologic hypoxia or physiologic foetal ripening? (Review). Early Hum Dev 2014;90:325-8.

12. Hutton EK, Thorpe J. Consequences of meconium stained amniotic fluid: what does the evidence tell us? Early Hum Dev 2014;90:333-9.

13. Beligere N, Rao R. Neurodevelopmental outcome of infants with meconium aspiration syndrome: report of a study and literature review. J Perinatol 2008;28 Suppl 3:S93-101.

14. Maimburg RD, Vaeth M. Perinatal risk factors and infantile autism. Acta Psychiatr Scand 2006;114:257-64.

15. Matsuishi T, Yamashita Y, Ohtani Y, Ornitz E, Kuriya N, Murakami Y, et al. Brief report: incidence of and risk factors for autistic disorder in neonatal intensive care unit survivors. J Autism Dev Disord 1999;29:161-6.

16. Piven J, Simon J, Chase GA, Wzorek M, Landa R, Gayle J, et al. The etiology of autism: pre-, peri- and neonatal factors. J Am Acad Child Adolesc Psychiatry 1993;32:1256-63.

17. Mrozek-Budzyn D, Majewska R, Kieltyka A. Prenatal, perinatal and neonatal risk factors for autism-study in Poland. Cent Eur J Med 2013;8:42430.

18. Bilder D, Pinborough-Zimmerman J, Miller J, McMahon W. Prenatal, perinatal, and neonatal factors associated with autism spectrum disorders. Pediatrics 2009; 123:1293-300.

19. Gregory SG, Anthopolos R, Osgood CE, Grotegut CA, Miranda ML. Association of autism with induced or augmented childbirth in North Carolina Birth Record (1990-1998) and Education Research (19972007) databases. JAMA Pediatr 2013;167:959-66. 
20. Wu DM, Wen X, Han XR, Wang S, Wang YJ, Shen M, et al. Relationship between neonatal vitamin $\mathrm{D}$ at birth and risk of autism spectrum disorders: the NBSIB study. J Bone Miner Res 2018;33:458-66.

21. Winkler-Schwartz A, Garfinkle J, Shevell MI. Autism spectrum disorder in a term birth neonatal intensive care unit population. Pediatr Neurol 2014; 51:776-80.

22. Gardener H, Spiegelman D, Buka SL. Perinatal and neonatal risk factors for autism: a comprehensive meta-analysis. Pediatrics 2011;128:344-55.

23. Dargaville PA. Respiratory support in meconium aspiration syndrome: a practical guide. Int J Pediatr 2012;2012:965159.

24. Beligere N, Rao R. Neurodevelopmental outcome of infants with meconium aspiration syndrome: report of a study and literature review. J Perinatol 2008;28 Suppl 3:S93-101.

25. Rossi AC, Prefumo F. Antepartum and intrapartum risk factors for neonatal hypoxic-ischemic encephalopathy: a systematic review with meta-analysis. Curr Opin Obstet Gynecol 2019;31:410-7.
26. Li C, Miao JK, Xu Y, Hua YY, Ma Q, Zhou LL, et al. Prenatal, perinatal and neonatal risk factors for perinatal arterial ischaemic stroke: a systematic review and meta-analysis. Eur J Neurol 2017;24:1006-15.

27. Salmaso N, Silbereis J, Komitova M, Mitchell P, Chapman K, Ment LR, et al. Environmental enrichment increases the GFAP+ stem cell pool and reverses hypoxia-induced cognitive deficits in juvenile mice. J Neurosci 2012;32:8930-9.

28. Rennie JM, Hagmann CF, Robertson NJ. Outcome after intrapartum hypoxic ischaemia at term. Semin Fetal Neonatal Med 2007;12:398-407.

How to cite this article: Jenabi E, Ayubi E, Khazaei S, Bashirian S, Khazaei M. Is meconium exposure associated with autism spectrum disorders in children? Clin Exp Pediatr 2021;64:341-6. https://doi.org/10.3345/cep.2020.01053 